services to support their staff. In the early stages of the pandemic there was a plethora of information outlining ways of managing the emotional impact of the pandemic. However, the information was not reaching the staff in an accessible way.

To address this we developed a simple guide to resilience and wellbeing for all staff. This joint project outlined different levels of support from informal peer led support and individual strategies, to formal referrals to bereavement or mental health services.

Outcomes and impact The guide received a national award from Skills for Care as an example of best practice. Following on from this work both Southampton CCG and West Hampshire CCG asked for the same guide to be sent to all care homes in Southampton city and West Hampshire.

\section{P-180 DEMENTIA, STRESS AND DISTRESS - SUPPORTING THE PALLIATIVE CARE WORKFORCE}

Julie Christie, Oonagh Thompson-Bradley, Tanya Clover. Hammond Care, London, UK

\subsection{6/spcare-2021-Hospice.196}

Background Behaviours and psychological symptoms of dementia (BPSD) are prevalent, complex, and distressing for people with dementia and caregivers. They are associated with additional stresses on people and systems, resulting in care packages breaking down, hospitalisations, caregiver burden, cognitive deterioration, psychotropic polypharmacy, and poor quality of life (Brodaty, Connors, Xu, et al., 2015). They are often the result of an unmet need such as pain. People living with dementia are often unable to self-identify or report pain and may express their discomfort through responses perceived as agitation and aggression. The correlation between behaviours and pain is known (Atee, Morris, Macfarlane, et al., 2021), yet more needs to be done to raise awareness with care staff, and to provide practical support as to what works. Aims To describe the characteristics of referrals to a health technology programme, developed in response to pandemic restrictions, which provides relationship-based support to care staff as they respond to behaviours, stress and distress.

Methods A pilot study evaluated the feasibility of the programme from June-September 2020. Referral data included demographic and clinical characteristics such as age and BPSD. Data were reported as descriptive statistics. All referrals received BPSD assessments using the Neuropsychiatric Inventory-Questionnaire (NPI-Q) (Kaufer, Cummings, Ketchel, et al., 2000).

Results Referral characteristics: Mean age: 84.3 years $(\mathrm{SD}=$ 6.7); Female: 67.9\%; Alzheimer's disease: 33.9\%; Primary cause of behaviour: delirium (32.1\%); pain (25.0\%); Mean total NPI-Q severity score: $12.8(\mathrm{SD}=6.5)$; Mean caregiver distress score: 15.7 ( $\mathrm{SD}=9.0)$; Referrals with $\geq 5$ neuropsychiatric symptoms: $67.9 \%$; $\geq 9$ symptoms: $14.3 \%$; Top three primary behaviours on referral: agitation, physical aggression, anxiety.

Conclusion BPSD were caused primarily by delirium and pain, both of which are prevalent but under-diagnosed in people with dementia (International Psychogeriatric Association, 2012). If care staff can access real-time support to mobilise assets and resources and build capacity, we can reduce the need for more costly health resources and the human costs of personal crisis (Macfarlane, Atee, Morris, et al., 2021).

\section{P-181 HOW TO ENSURE THAT THOSE WHO DELIVER CARE ARE THEMSELVES WELL LOOKED AFTER- THRIVE OR SURVIVE!}

Liz Matthews. Ashgate Hospicecare, Chesterfield, UK

\subsection{6/spcare-2021-Hospice. 197}

Background A pilot scheme was delivered during the COVID19 pandemic to support staff at a local hospital to cope with the unprecedented traumatic situation. This highlighted shortfalls and the need to promote systemic change in wellbeing provision, to encourage a more robust and accessible programme of support for healthcare professionals in multi-disciplinary organisations working with grief, loss, and trauma pre/ post bereavement.

Aims To develop multidisciplinary tailor-made support for healthcare professionals, embracing cultural diversity, self-sustaining resilience, and empowerment. This support will incorporate various approaches and will be developed to meet presenting needs. It will aim to improve communication and develop a greater understanding between staff teams and organisations, encouraging working in partnership. It will promote transparency and openness as opportunities for learning and improvement rather than blame and punishment. It will lessen work-related stress and anxiety, create a happier and more compassionate environment, which in turn will improve quality of care.

Methods Review of literature. Feedback analysis. Internal consultation via multidisciplinary steering group meetings. Liaise with wellbeing department to work in collaboration. Consultation with relevant local organisations. Secure premises. Funding application. Recruit staff. Recruit and train specialist volunteers to provide therapeutic support. Continuous service review and evaluation using core assessment outcomes.

Results

- Increased coping strategies, emotional resilience, and mental health, measured by Core 10 psychological assessment.

- Increased peer and multidisciplinary support verified by group activity attendance and staff feedback.

- Improved communication channels.

- Staff training to deliver reflective practice and debriefs.

- Development of a specialist volunteer team

- A programme of workshops and therapeutic groups delivered to meet staff need.

Conclusion Evaluation showed increased awareness of the importance of self-care and peer support amongst healthcare professionals. Greater autonomy of support enabled a willingness to engage in wellbeing provision leading to additional coping strategies and resilience.

\section{YOU CAN'T POUR FROM AN EMPTY JUG}

Sarah Fathers, Mary Mooney . Ardgowan Hospice, Greenock, UK

\subsection{6/spcare-2021-Hospice. 198}

Background In the earliest stages of the COVID-19 pandemic, Inverclyde was particularly badly impacted. Ardgowan Hospice felt strongly that, as well as supporting the local community, it didn't want those involved in supporting and caring for others during this unprecedented time to be overlooked. 